\title{
Microleakage of Silorane-based Resin Composite in Comparison with Methacrylate-based Composite in Class II Open Sandwich Restorations: An in vitro Study
}

\author{
Mithra N Hegde, Nanditha Hegde, Nidarsh D Hegde
}

\begin{abstract}
Introduction: This in vitro study aimed to evaluate the effect of different monomer systems on microleakage in class II composite open sandwich restorations with and without a separating agent placed between the nanofilled resin modified glass ionomer and the resin.
\end{abstract}

Materials and methods: Class II cavity preparations were prepared in maxillary premolars $(n=240)$ and the cervical limit of the proximal box was below the cementoenamel junction. Group 1 was restored with silorane-based composite and group II was restored with methacrylate-based composite. All specimens were subjected to thermocycling and examined for microleakage after in $50 \%$ silver nitrate solution. The recorded results of dye penetration were statistically analyzed using Chisquare test.

Results: Methacrylate-based composite (Filtek Z 350, 3M, St Paul, MN, USA) exhibited the highest dye penetration and silorane-based composite (Filtek P90 Low Shrink Posterior Restorative, 3M, St Paul, MN, USA) showed the lowest dye penetration among the restorative materials. However, specimens with separating agent showed almost no leakage between the RMGIC and tooth interface.

Conclusion: Forces of polymerization shrinkage are stronger than the bond between glass ionomer cement and tooth interface and the use of silorane technology in open sandwich restoration will decrease microleakage and improve clinical performance.

Keywords: Open sandwich restoration, Silorane, Methacrylate, Microleakage.

How to cite this article: Hegde MN, Hegde N, Hegde ND. Microleakage of Silorane-based Resin Composite in Comparison with Methacrylate-based Composite in Class II Open Sandwich Restorations: An in vitro Study. World J Dent 2012;3(2):145-149.

Source of support: Nil

Conflict of interest: None declared

\section{INTRODUCTION}

Resistance to microleakage of any restorative material and its behavior with respect to marginal adaptation is of great importance. ${ }^{1,2}$ Greater microleakage has been reported below cementoenamel junction than above it especially in posterior resin preparations. Increased polymerization shrinkage of composite material leads to poor marginal adaptation. $^{3-6}$

None of the available resin restoration system have been reported to provide complete adhesion to cementodentinal walls. With the use of glass ionomer various advantages were reported such as bonding chemically to tooth structure and micromechanically to composites, biological acceptance, fluoride release, reduced microleakage and marginal integrity. $^{7,8}$

The main adverse effect of methacrylate polymerization reaction is volumetric shrinkage. One of the strategies for controlling polymerization contraction stress is using a novel monomer system that has decreased polymerization shrinkage, such as siloranes. Although siloranes can form a strong bond with identical material (siloranes), its capacity to form a bond between a dissimilar resin systems like with resin-modified glass ionomer is not completely known. ${ }^{9}$

Thus, there is a need for studying the effect of different monomer systems, such as methacrylates and siloranes on microleakage in class II composite open sandwich restorations.

\section{MATERIALS AND METHODS}

Two hundred and forty freshly extracted caries-free human maxillary premolars (for orthodontic purposes; within a period of 30 days) were collected from the Department of Oral and Maxillofacial Surgery, AB Shetty Memorial Institute of Dental Sciences, and stored in saline at room temperature $\left(28 \pm 2{ }^{\circ} \mathrm{C}\right)$.

Restorative procedures: All the teeth received were cleaned with slurry of pumice washed and dried. A template was prepared and each specimen tooth to be prepared and restored was pressed in the space left on the template with adjacent teeth for placement of tofflemire matrix which allowed the building of the proximal wall.

Specimen preparation: Class II mesio-occlusal cavity preparations using \#245 carbide burs (D-Flex, Germany, CE8120) in a high speed handpiece with copious water cooling were prepared in all the selected teeth and for every five preparations a new bur was used. All the preparations were performed by the same operator. The operator performed these procedures under $2.5 \mathrm{x}$ magnification loupe (Heine, HOSPEQ, Inc, USA).

The occlusal dimension of the cavity approximately twothirds of the intercuspal dimension and the cervical limit of the proximal box is $1 \mathrm{~mm}$ below the cementoenamel 
junction. Floor of the proximal box was rinsed and dried with endodontic paper cone avoiding any excessive dehydration. The teeth will be randomly placed into two main groups $(n=120)$, depending on the restorative material used. Each group was further subdivided into two subgroups $(n=60)$.

Group 1 (Restored with silorane-based composite) $(\mathrm{n}=120)$.

Group 2 (Restored with methacrylate-based composite) $(\mathrm{n}=120)$.

Open sandwich technique: A custom-made matrix band (Fig. 1) which consisted of a metal matrix band fused to mylar matrix band and a reflective wedge (3M, St Paul, MN, USA) were inserted and adapted on the surface of the tooth using tofflemire matrix retainer which allowed the building of the proximal wall. The nanofilled RMGIC (Ketac N100) primer (3M, St Paul, MN, USA) was applied; air dried and light cured as per manufacturer's instructions with a quartz-tungsten-halogen unit (QLF 75 curing light, Model 502, Dentsply Caulk, Milford, CT, USA) with a light intensity of $500 \mathrm{~mW} / \mathrm{cm}^{2}$ as calculated by using a light optimeter (Optiflux radiometer, Kerr Sybron dental specialities, Middleton,WI, USA), and then the two pastes are mixed and inserted into the proximal cavity extending cervically to the periphery of the proximal cavity leaving a layer of $2 \mathrm{~mm}$ in depth.

The cavities were restored with composite in oblique increments not greater than $2 \mathrm{~mm}$ in thickness and light cured using three-site technique ${ }^{10}$ (Fig. 2) according to manufacturer's instructions through reflective wedges.

The group B subgroups of each group received four layers of copal varnish (Harvard Dental International GmbH, Hoppegarten, Germany) to the surface of the gingival seat of the resin modified glass ionomer and did not receive any

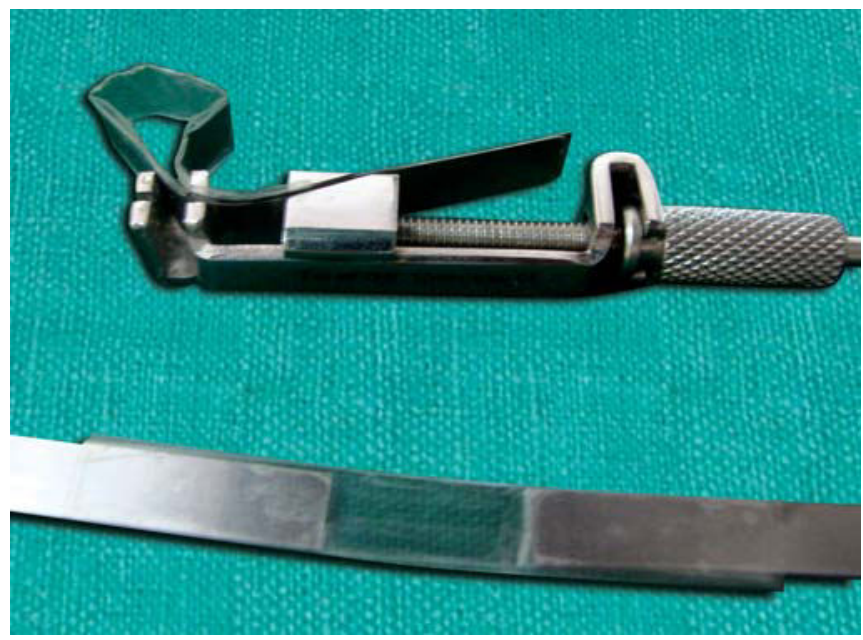

Fig. 1: Custom-made matrix band dentin-bonding agent on the gingival seat before the placement of the composite.

Thus, resulting in the following four groups:

- Group 1A: Light curing resin-modified glass ionomer sandwiched with silorane-based composite.

- Group 1B: Light curing resin-modified glass ionomer sandwiched with silorane-based composite sandwiched with a separating agent placed between them.

- Group IIA: Light curing resin-modified glass ionomer sandwiched with methacrylate-based composite.

- Group IIB: Light curing resin-modified glass ionomer sandwiched with methacrylate-based composite a separating agent placed between them.

Thermocycling and microleakage test: The restored teeth were then stored for 30 days at $37^{\circ} \mathrm{C}$ in distilled water. All specimens were then subjected to thermocycling at $5^{\circ} \mathrm{C}$ and $55^{\circ} \mathrm{C}$ for 3000 cycles, with 30 seconds dwell time at each bath. The teeth were then immersed in $50 \%$ silver nitrate (Rankem, RFCL, India) solution for 4 hours. Following immersion the teeth were washed thoroughly with distilled water and immersed in developing solution for 6 hours under fluorescent light.

Microleakage evaluation: All the teeth were sectioned midsagitally in the mesial distal plane using diamond disks (Isomet, Buehler, IL, USA) at slow speed. The cervical margin interface was examined under the stereomicroscope (Lawrence and Mayo, Labomed Zoomer) at $30 \times$ magnification for evidence of dye penetration. The maximum degree of dye penetration was recorded for each section and the dye penetration was scored on a nonparametric scale from zero to four based on the Saboia et al. Criteria for microleakage analysis are described as follows: ${ }^{11}$

\section{Scoring criteria}

- 0 : No dye penetration.

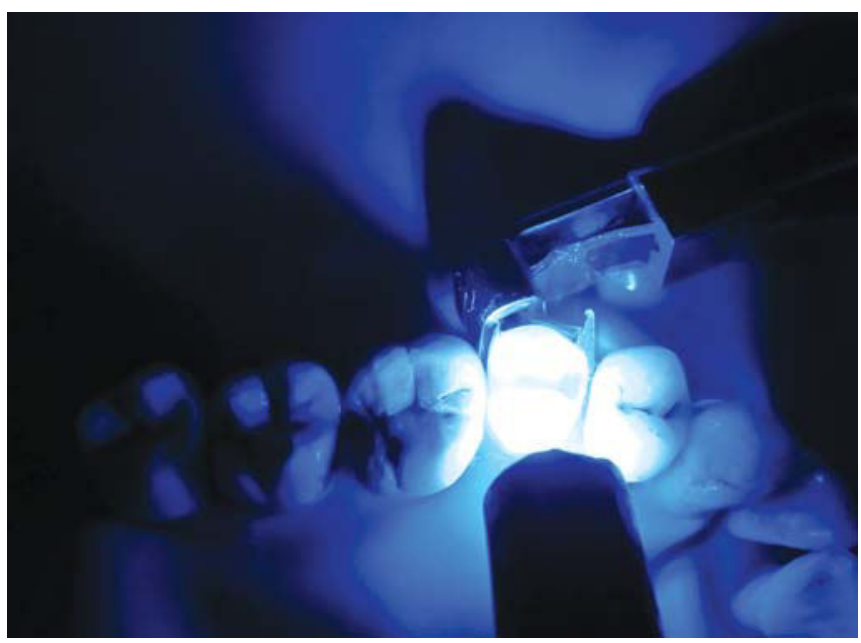

Fig. 2: Three-site light curing technique 


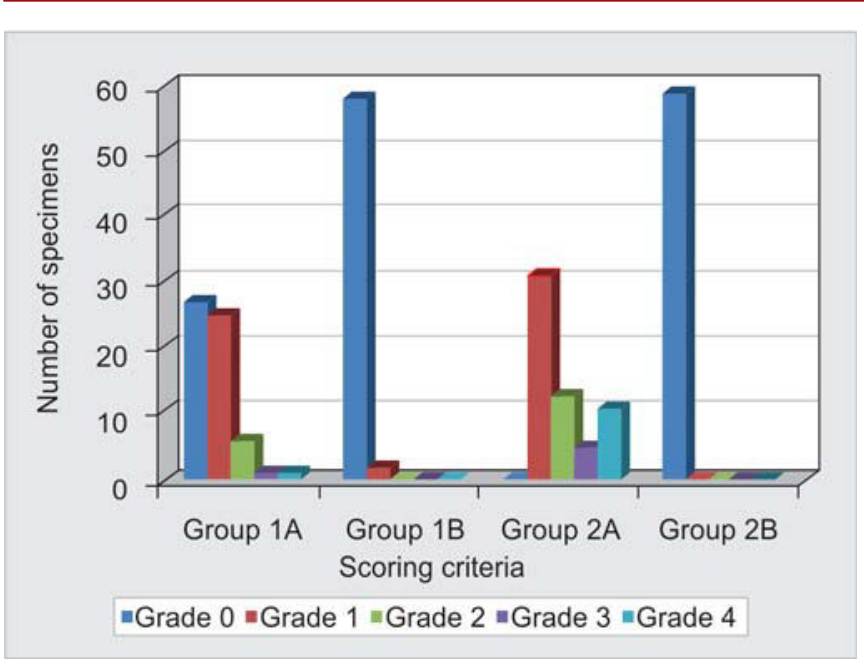

Fig. 3: The comparison of mean microleakage of the four experimental groups

- 1: Dye penetration upto $1 / 3$ rd of the length of the gingival wall.

- 2: Dye penetration from $1 / 3$ rd to $2 / 3$ rd of the length of the gingival wall.

- 3: Dye penetration greater than score 2 but not including the axial wall.

- 4: Dye penetration with penetration spreading along the axial wall.

Statistical methods: The results were tabulated and the recorded results of dye penetration were statistically analyzed using Chi-square test. SPSS (Statistical Package for Social Science) software version 15 was used. Level of significance was set at $\mathrm{p}=0.05$. $\mathrm{p}$ value $<0.05$ was considered to be statistically significant.

Bar chart was used for statistical representation (Fig. 3).

\section{RESULTS}

The computed value of $p<0.05$ indicates statistically significant difference between the groups under study. Of the two products evaluated, methacrylate-based composite (Filtek Z 350) exhibited the highest dye penetration and silorane-based composite (Filtek P90 Low Shrink Posterior Restorative) showed the lowest dye penetration among the restorative materials. Further the data was subjected to the Chi-square test to determine the intergroup comparison. There is statistical significance between group $1 \mathrm{~A}$ and $1 \mathrm{~B}$ and between group 2A and 2B. Specimens with separating agent showed almost no leakage between the resin-modified glass ionomer cement and tooth interface.

\section{DISCUSSION}

The apparent inability to seal margins below the CEJ margins has always been a vexing and lasting obstacle.
The existence of an outer layer which is around 200 to 300 $\mu \mathrm{m}$ thick at the cementoenamel junction margin, not identifiable as sound dentin and covered by a thin layer of cementum; does not form a well-defined hybrid layer and the presence of resin tags were rare and was considered hypomineralized and hyperorganic, and hence may effect the bonding. ${ }^{12-14}$ More research related to this critical area is needed so as to predict more effectible bonding.

Debonding at the restorative tooth interface is caused by polymerization shrinkage stress in the resin matrix which may result in microleakage further recurrent caries and finally pulpitis. ${ }^{15-17}$ Several alternative clinical techniques have been introduced to reduce the stress-related problems in class II cavities, among these composite laminated RMGIC or sandwich technique has proven to be effective. Its effectiveness is due to the intrinsic porosity of the cement leading to reduced stress. ${ }^{18,19}$

Use of flowable composites is a common clinical technique thought to reduce microleakage when placed with composite restorations. However, due to the significant amount of unfilled resin found in flowables relative to filler load this potential benefit as stress reducing liners could be offset by increased polymerization shrinkage stress. ${ }^{16}$ Hence, flowable composites were not considered an ideal for open sandwich technique in the current study.

Clinical evaluation has shown a high failure rate with the use of conventional GICs in open sandwich restorations due to partial or total dissolution of the GIC or fracture of the restorations. RMGICs which have high degree of elastic deformation during the early stage of setting can relieve contraction stresses by acting as an elastic buffer. ${ }^{20-22}$

With the incorporation of nanotechnology RMGICs allows highly packed filler composition of which approximately two-third are nanofillers. ${ }^{23}$ In this study the nanofilled type of glass ionomer show the lowest microleakage scores. This may be explained by the fact that the nanostructure of the nanofilled glass ionomer type allowed for excellent wetting and adaptability to the tooth surface, hence enhancing the chemical bonding.

In an attempt to solve the shrinkage problem of direct resin composite restorations, a new category of resin matrix was developed based on ring opening monomers. ${ }^{24}$ Silorane P90 is a microhybrid and is considered to have combined two key advantages of the individual components: Low polymerization shrinkage due to the ring opening oxirane monomer and increased hydrophobicity due to presence of the siloxane species so, it results in reduced water uptake and related phenomena. ${ }^{15}$

In order to reduce contraction stresses, the oblique incremental technique has been advocated for the placement of the composite, which lowers the configuration factor. ${ }^{22}$ 
Halogen curing light devices are the most commonly utilized light sources hence used in the present study. ${ }^{15}$ Sensei et al examined different types of light-generating units and found no difference in dye penetration between varying types of units. $^{15}$

A majority of studies demonstrate the effectiveness of the three-site technique and the idea behind this technique takes advantage of contraction toward the light theory (Lutz et al 1986; Lutz et al 1991; Lutz, Krejci and Barbakow, 1992). ${ }^{17}$ In the present study, use of reflective wedge and modified transparent matrix may have lead to the attenuation of the light intensity thus decreasing the contraction stresses and improving the adaptation. ${ }^{17}$ Kays et al $(1991)^{25}$ showed that polymerization is dependent on the matrix band and wand positioning during polymerization. Curing light intensity in the most apical portion of class II cavity with cervical margins in cementum may not be sufficient if a metal matrix is used. ${ }^{15}$ Hence, in this study a custom-made matrix was used which was a combination of metal matrix and transparent matrix which provides adequate adaptation of the matrix around the tooth structure as well as adequate curing light intensity in the most apical portion of class II cavity for optimum polymerization.

In the present study, specimens with copal varnish between the resin-modified glass ionomer cement and composite resin showed almost no leakage. These results indicate that when the mechanical bond between the composite and the resin-modified glass ionomer is prevented, there is no separation at the resin-modified glass ionomer cementum interface, and thus no microleakage. When resin modified glass ionomer is allowed to bond to the resin, it appears that the polymerization shrinkage of the setting resin causes the debonding at the resin-modified glass ionmer cementum interface.

The results of this study show that the low-shrink composite restorative material had significantly less microleakage. This is in accordance Bagis, ${ }^{26}$ Yamazaki $^{27}$ and Plain ${ }^{28}$ who proved that the microleakage of silorane is lower than that of methacrylate-based composite. Our study does not agree with Ernst ${ }^{29}$ who proved that the microleakage of teeth restored with silorane was similar to others restored with methacrylate composite.

We found that resin-modified light-cured glass ionomer cement when used under a composite restoration placed below gingival cementum/dentin margin had better sealing performance. Both the resin composite materials used in this study had some degree of leakage. Hence, despite advances that have been made, many clinical and material limitations have restricted their use as a posterior restorative material.

\section{ACKNOWLEDGMENT}

We acknowledge the financial support provided by ICMR New Delhi for this research.

\section{REFERENCES}

1. Anthony HL Tjan, James R Dunn. Microleakage at gingival dentin margins of class 5 composite restorations lined with light cured glass ionomer cement. JADA 1990;12:705-10.

2. Chin TH, Martin JT. Adhesion of composite resin to etched glass ionomer cement. Aust Dent Jour 1988;33(2):87-90.

3. David JE, Fen HW. Polymerization shrinkage of posterior composite resins and its possible influnce on postoperative sensitivity. Quint Int 1986;17(2):103-11.

4. Edward L Welsh, John H Hembree. Microleakage at the gingival wall with four class 5 anterior restorative material. JPD 1985; 54(3):70-72.

5. Santini A, Plasschaert AJ, Mitchell S. Marginal leakage of filled dentin adhesives used with wet and dry bonding techniques. Am J Dent 2000;13(2):93-97.

6. Santini A, Plasschaert AJ, Mitchell S. Effect of composite resin placement techniques on the microleakage of two self-etching dentin-bonding agents. Am J Dent 2001;14(3):132-36.

7. Farah CS, Orton VG, Collard SM. Shear bond strength of chemical and light cured glass ionomer cements bonded to resin composites. Aust Dent Jour 1998;43(2):81-86

8. Me Lean JW, Prosser HJ, Wilson AD. The use of glass ionomer cements in bonding composite resins to dentin. Brit Dent J 1985;158:410-14.

9. Sillas Duarte Jrjin - Ho Phark. Nanoleakage, ultramorphological characteristics, and microtensile bond strengths of a new low shrinkage composite to dentin after artificial aging. Dental Materials 2009;25:589-600.

10. Carel 1. Davidson. Glass-Ionomer bases under posterior composites. Journal of Esthetic and Restorative Dentistry 1994;6:223-26

11. Rossomando KJ, Wendt SL Jr. Thermocycling and dwell times in microleakage evaluation for bonded restorations. Dent Mater 1995;11(1):47-51.

12. Beznos C. Microleakage at the cervical margin of composite class II cavities with different restorative techniques. Oper Dent 2001;26:60-69.

13. Ferrari M, Davidson CL. Sealing performance of Scotchbond Multipurpose-Z100 in Class II restorations. American Journal of Dentistry 1996;9(4):145-49.

14. Ferrari M, Mason PN, Fabianelli A, Cagidiaco MC, Kugel G, Davidson CL. Influence of tissue characteristics at margins on leakage of Class II indirect porcelain restorations. American Journal of Dentistry 1999;12:134-42.

15. Dietrich T, Losche GM, Roulet JF. Dietrich T, Losche GM, Roulet JF. Marginal adaptation of direct composite and sandwich restorations in Class II cavities with cervical margins in dentine. Journal of Dentistry 1999;27:119-28.

16. Yip KH, Poon BK, Chu FC, Kong FY, Smales RJ. Clinical evaluation of packable and conventional hybrid resin-based composite for posterior restorations in permanent teeth: Result at 12 months. JADA 2003;134:1581-89.

17. Lindberg A, van Dijken JW, Lindberg M. Nine-year evaluation of a polyacid-modified resin composite/resin composite open sandwich technique in Class II cavities. J Dent. 2007;35(2): 124-29. 
18. Davidson CL, Feilzer AJ. Polymerization shrinkage and polymerization shrinkage stress in polymer-based restoratives. Journal of Dentistry 1997;25:435-40.

19. Loguercio AD, Alessandra R, Mazzocco KC, Dias AL, Busato AL, Singer Jda M, Rosa P. Microleakage in Class II composite resin restorations: Total bonding and open sandwich technique. Journal of Adhesive Dentistry 2002;4(2):137-44.

20. Van Dijken JWV, Kieri C, Carlén M. Longevity of extensive Class II open-sandwich restorations with resin-modified glassionomer cement. J Dent Res 1999;78(7):1319-25.

21. Van Dijken JW. A 6-year evaluation of a direct composite resin inlay/onlay system and glass ionomer cement-composite resin sandwich restorations. Acta Odontol Scand 1994;52(6):368-76.

22. Tolidis K, Nobecourt A, Randall RC. Effect of a resin-modified glass ionomer liner on volumetric polymerization shrinkage of various composites. Dent Mater 1998;14(6):417-23.

23. Coutinho E, Cardoso MV, De Munck J, Neves AA, Van Landuyt KL, Poitevin A, et al. Bonding effectiveness and interfacial characterization of a nano-filled resin-modified glass-ionomer. Dent Mater 2009;25(11):1347-57.

24. Weinmann W, Thalacker C, Guggenberger R. Siloranes in dental composites. Dent Mater 2005;21(1):68-74.

25. Kays BT, Sneed WD, Nuckles DB. Microhardness of Class II composite resin restorations with different matrices and light positions. J Prosthet Dent 1991 Apr;65(4):487-90.

26. Bagis $\mathrm{YH}$, Baltacioglu IH. Comparing microleakage and layering methods of silorane based resin composite in wide Class II MOD cavities. Operative Dentistry 2009;34-5:578-85.
27. Yamazaki, Bedran-Ruso AKB, Pereira PNR. Microleakage evaluation of a new low-shrinkage composite restorative material. Operative Dentistry 2006;31-6:670-76.

28. Plain WM, Fleming GJ, Nathwani H, Burke FJ, Randall RC. In vitro cuspal deflection and microleakage of maxillary premolars restored with novel low shrink dental composites. Dental Materials 2005;21:324-35.

29. Ernst CP, Galler P, Willershausen B, Haller B. Marginal integrity of class $V$ restorations: SEM versus dye penetration. Dent Mater 2008;24:319-27.

\section{ABOUT THE AUTHORS}

\section{Mithra N Hegde}

Senior Professor and Head, Department of Conservative Dentistry and Endodontics, AB Shetty Memorial Institute of Dental Sciences Bengaluru, Karnataka, India

\section{Nanditha Hegde (Corresponding Author)}

Postgraduate Student, Department of Conservative Dentistry and Endodontics, AB Shetty Memorial Institute of Dental Sciences Bengaluru, Karnataka, India, e-mail: nanditahegde@ hotmail.com

\section{Nidarsh D Hegde}

Senior Professor, Department of Oral and Maxillofacial Surgery AB Shetty Memorial Institute of Dental Sciences, Bengaluru, Karnataka India 
